\title{
Simulation of Tourism Supply Chain Collaborative Demand Forecast
}

\author{
Hao-yuan Zhang, Hua Bai \\ Shenzhen Tourism College, Jinan University, China
}

\begin{abstract}
Supply chain collaborative demand forecast has gained more and more attention. In particular, for tourism supply chain (TSC), due to the high market volatility and uncertainty, travel agencies, scenic spots, hotels and other tourism enterprises in the tourism supply chain need a tight collaboration to enhance sharing of information and resources. This paper proposes a simulation method of tourism supply chain collaborative demand forecast to analyze the effectiveness and gain insight.
\end{abstract}

Keywords - Tourism supply chain (TSC), Collaborative demand forecast, simulation.

\section{旅游供应链协同需求预测仿真}

\author{
张浩元 白华 \\ 暨南大学深圳旅游学院电子商务系, 深圳, 广东, 中国
}

摘 要 供应链的协同需求预测愈来愈受到重视, 尤其是旅游供应链。旅游需求呈现出多样性和不确定性, 以及易受政治和经济环 境的影响, 旅游供应链上各个结点企业间的协同显得越发重要。旅游供应链的协同需求预测可以使旅行社、景点等结点企业信息和资 源共享, 能更精确和动态地了解游客需求从而改进旅游产品, 更好地适应外界环境变化对旅游需求的影响。本文建立了一种旅游供应 链协同需求预测的流程仿真方法, 并进行了探讨。

关键词 旅游供应链, 协同需求预测, 仿真

\section{1. 引言}

在全球信息化和市场全球化, 科技加速变革的背景 下，各行业之间的竞争日趋激烈。不断缩短的交货期和产 品生命周期、提高质量、降低成本、改进服务、产品制造 与市场的国际化、动态需求、动态组织和动态管理共同构 成企业动态和不确定的生产环境。然而像 2012年钓鱼岛事 件的发生, 造成日本旅游业大幅度影响, 国内外旅游供应 链都有不同程度的损失, 因此旅游供应链的精准需求预测 则显得越发重要[1][2]。

旅游供应链的协同需求预测涉及大量的实时信息, 跨 越多个不同行业的节点企业, 缺乏标准化和流程的规范 化。对协同需求预测模型的建模、分析和验证, 需要一个 有力的仿真工具。着色 Petri 网(Colored Petri-nets, CPN) 是 用来分析包含协同在内众多领域问题的建模技术，它非常
适合诸如供应链之类的离散事件系统建模与分析 [3][4]。 本文选取了 CPN Tools[5]建立了一种基于 CPN 的旅游供应 链协同需求预测仿真系统, 并通过案例分析验证了其可行 性和有效性。

\section{2. 旅游供应链协同需求预测流程}

\section{1 框架构建}

本文设计了基于旅游供应链的协同需求预测流程框 架, 如图 1 所示。 


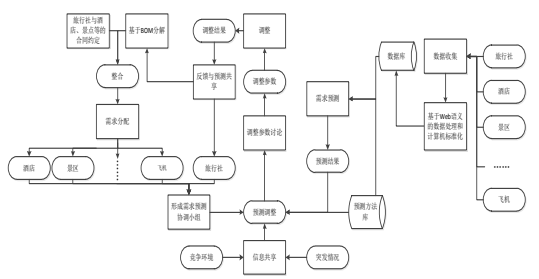

图 1 基于旅游供应链的协同需求预测流程框架

\section{2 运作流程}

(1) 获取旅行社、酒店、景点、飞机等旅游供应链上 的相关信息, 减少信息的分散度, 集成信息将有利于做出 更精准的预测和决策。从旅行社获取旅行产品价格数据、 成团数、新旅游产品信息、产品组成、变更产品信息, 促 销活动等相关数据; 同样获取供应链上其他节点的相应信 息, 并对供应链外的竞争对手进行研究, 获取其他企业的 竞争策略、市场/产品/服务等的信息, 兼顾环境与社会政策 等信息。

(2) 将获取的数据和信息转化成计算机数据。进行基 于语义 Web 的数据处理和计算机标准化, 产生可以使用的 统一的数据格式, 然后存进协同需求预测数据库。

(3) 由旅游供应链上的不同成员共同组成一个协同需 求预测小组, 可以对预测结果、预测方法、例行事务的决 策、竞争对手的战略和突发情况等进行协调控制。协同需 求预测小组根据多种组合型预测模型和方法、历史数据等 进行分析, 对其预测结果判断其合理性。如果合理则转入 下一步。如果需要进行调整, 则征求旅行社、酒店、景点 等企业的意见，各节点进行协商后做出必要的调整。同时， 时刻监测竞争对手、社会政策和天然环境等的变化, 对竞 争对手在策略以及企业营销方面的行为进行分析, 对天气 状况、政策环境的经济变化等进行敏感性分析, 如果发生 调整, 征求供应链上各方对应的看法与意见, 然后重新修 正需求预测参数。

(4) 对旅游产品所需要的床位、景区门票等进行分配。 在对旅游产品进行预测后, 需要将预测结果信息分享给旅 游供应链中的成员, 生成旅客需求。本仿真提出的方法是 基于 BOM (Bill of Materials) 结合旅行社与其他成员签订的 合同进行需求分解。假定旅游产品 $\mathrm{A}$ 的预测结果为 $\mathrm{FA}$, 其 旅游需求组成分别为 $\{\mathrm{D} 1, \cdots, \mathrm{Dm}\}, \mathrm{BOM}$ 清单为 $\{\mathrm{BD} 1$, $\mathrm{BD} 2, \cdots, \mathrm{BDm}\}$, 且基于签订的合同有若干供应商（包含 $\mathrm{n}$ 处酒店与 $\mathrm{n}$ 个景点等） $\{\mathrm{S} 1, \mathrm{~S} 2, \cdots, \mathrm{Sn}\}$ 提供不同的比例数 量。在此因为旅游产品固有的特殊性是没有库存的, 所以 忽略库存的影响。

本文用比例进行描述如下:

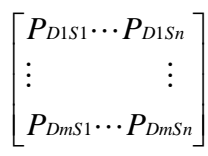

需求组成 Di 对各供应商的需求结果如式 (1) 所示:

$$
\begin{aligned}
& F(D i)=\left(F_{D} \times B_{D i}\right) \times\left(P_{D i S 1} \quad P_{D i S 2} \quad \cdots P_{D i S n}\right) \\
& =\left[\begin{array}{llll}
\left(F_{D} B_{D i} P_{D i S 1}\right) & \left(F_{D} B_{D i} P_{D i S 2}\right) & \cdots & \left(F_{D} B_{D i} P_{D i} S_{n}\right)
\end{array}\right]
\end{aligned}
$$

(5) 对旅客的需求计划进行实施后, 再将旅行社、酒 店、景区等的现实需求返回协同需求预测小组, 把预测的 需求与现实需求进行对比, 之后将结果与各个供应链上的 成员进行回馈和征集意见，对仿真的协同需求预测方法的 调用和管理进行必要修正, 调整预测参数从而提高预测精 度, 再返回第 (3) 步, 重新循环过程, 直到意见最小化以 及下次预测需求出现为止。

\section{3. 基于 $\mathrm{CPN}$ 的仿真构建}

\subsection{CPN 和 CPN Tools}

经典Petri 网是简单的过程仿真, 包含四个基本元素: 库所(Place) 、变迁(Transition) 、有向弧(Arc) 以及令牌 (Token)。

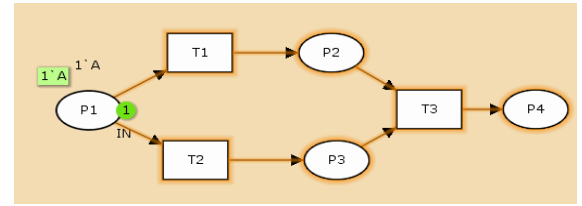

图 2 Petri 网的基本元素

Petri 网通过研究仿真模型的结构和动态行为, 模拟整 个系统组织网络中可能发生的状态变化, 以此突出变化之 间的关系。其具有强大的描述能力, 但对现实的系统构建 来说, 拥有性能良好的建模、仿真能力和模型检验能力的 工具对系统结构的检验才具有应用价值。而且 Petri 网多个 变迁争夺一个令牌使得冲突的存在, 造成了不确定性, 其 随机性对需求预测有良好的仿真作用。CPN Tools 是由丹麦 阿尔胡斯大学的 CPN 小组研制的建模软件, 是一个可以用 于建模、仿真和对高级 Petri 网进行分析模拟的重要工具, 它的出现使 Petri 网在现实系统中的模拟应用越来越广泛。

\section{2 参数与变量的定义}

通过对运作流程的分析结合 CPN Tools 的规则, 对行 为颜色集 (colset)与数据变量 (var) 的定义如图 3 所示。

\section{3 基于 $\mathrm{CPN}$ 的的仿真}

因为模拟的需要, 在此仅以旅行社、酒店 1 、酒店 2 
和景区售票作为旅游供应链的组成部分。基于旅游供应链 的协同需求预测流程框架, 结合其运作流程, 充分利用如 绑定 (Binding) 和门卫 (Guard) 用来条件输出控制等 CPN Tools 的特点进行建模, 在没有令牌的情况下, 效果如图 4 所示。

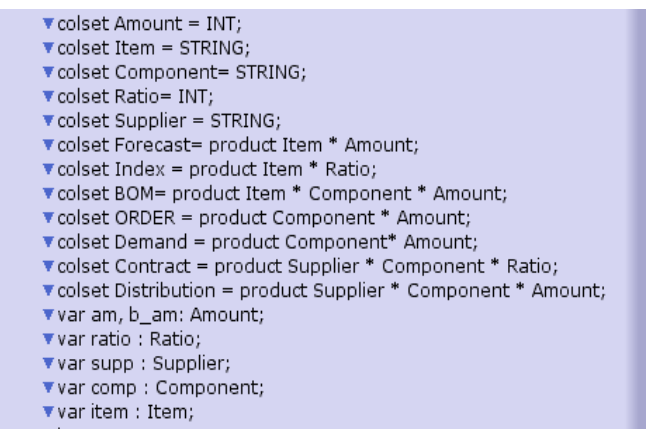

图 3 参数与变量的定义 $(\mathrm{CPN})$

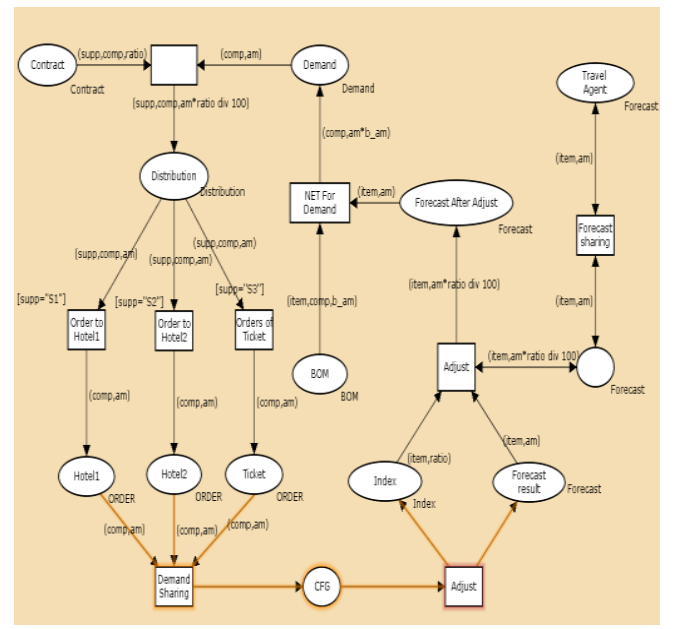

图 4 基于 $\mathrm{CPN}$ 的旅游供应链的协同需求预测流程仿真

\section{4. 案例应用分析}

\section{1 案例数据模拟}

为使仿真简单化且符合现实, 此文的仿真初始条件设 定为: 只有三种旅游产品 $\mathrm{A} 、 \mathrm{~B}$ 和 $\mathrm{C} 。 \mathrm{~A}$ 是单人景区两日一 夜游, $\mathrm{B}$ 是情侣双人景区两日一夜游, $\mathrm{C}$ 是学生双人景区两 日一夜游。其中 $\mathrm{B} 1$ 指单人床, $\mathrm{B} 2$ 指双人床; $\mathrm{T} 1$ 指普通景 区门票, T2 指学生景区票。详情如下 BOM 表:

表 1 旅游产品 BOM 表

\begin{tabular}{|c|c|c|c|c|}
\hline 旅游产品 & 床位类型 & 数量 & 景区门票 & 数量 \\
\hline $\mathrm{A}$ & $\mathrm{B} 1$ & 1 & $\mathrm{~T} 1$ & 1 \\
\hline $\mathrm{B}$ & $\mathrm{B} 2$ & 1 & $\mathrm{~T} 1$ & 2 \\
\hline $\mathrm{C}$ & $\mathrm{B} 1$ & 2 & $\mathrm{~T} 2$ & 2 \\
\hline
\end{tabular}

表 2 供应商合约表

\begin{tabular}{|c|c|c|c|c|}
\hline 供应商 & 供应产品 1 & 比例\% & 供应产品 2 & 比例\% \\
\hline Hotel1 & B1 & 30 & B2 & 60 \\
\hline Hotel2 & B1 & 70 & B2 & 40 \\
\hline Ticket & T1 & 100 & T2 & 100 \\
\hline
\end{tabular}

假设基于历史数据和旅游供应链的基本数据中研究出 的预测已知: 需要旅游产品 A100 份、旅游产品 B400 份、 旅游产品 $\mathrm{C} 200$ 份;

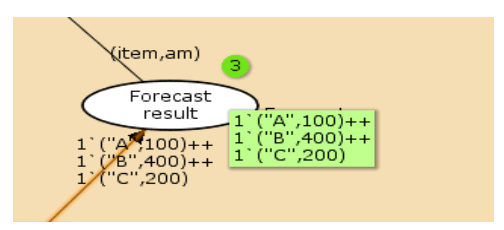

图 5 假设需求预测结果 $(\mathrm{CPN})$

需求预测协调小组已将对预测结果所做出的调整提出 了修改参数, 即是下图“Index”的值: 在 3 种旅游产品中: 选择产品 $\mathrm{A}$ 的需求概率是 $140 \%$, 选择产品 $\mathrm{B}$ 的概率是 $90 \%$, 选择产品 $\mathrm{C}$ 的概率是 $70 \%$ 。

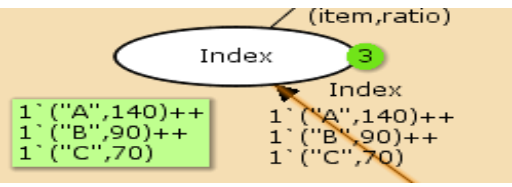

图 6 假设修改参数 $(\mathrm{CPN})$

在令牌充足的情况下, 需求预测理想状况根据式(2)和 式 (3) 计算结果为表 3 和表 4 所示:

\section{Item $=$ amount $*$ ratio}

Item=amount*ratio*box_amount*ratio

表 3 旅行社在理想状况下的需求预测结果

\begin{tabular}{|c|c|c|c|}
\hline & 旅游产品 $\mathrm{A}$ & 旅游产品 $\mathrm{B}$ & 旅游产品 $\mathrm{C}$ \\
\hline 旅行社 & 140 & 360 & 140 \\
\hline
\end{tabular}

表 4 供应商在理想状况下的需求预测结果

\begin{tabular}{|c|c|c|c|c|}
\hline & B1 & B2 & T1 & T2 \\
\hline 酒店 1 & 126 & 216 & & \\
\hline 酒店 2 & 294 & 144 & & \\
\hline 景区 & & & 860 & 280 \\
\hline
\end{tabular}




\section{2 案例仿真}

整合案例的初始模拟数据, 通过对令牌的修改进行仿 真, 仿真的初始图如下:

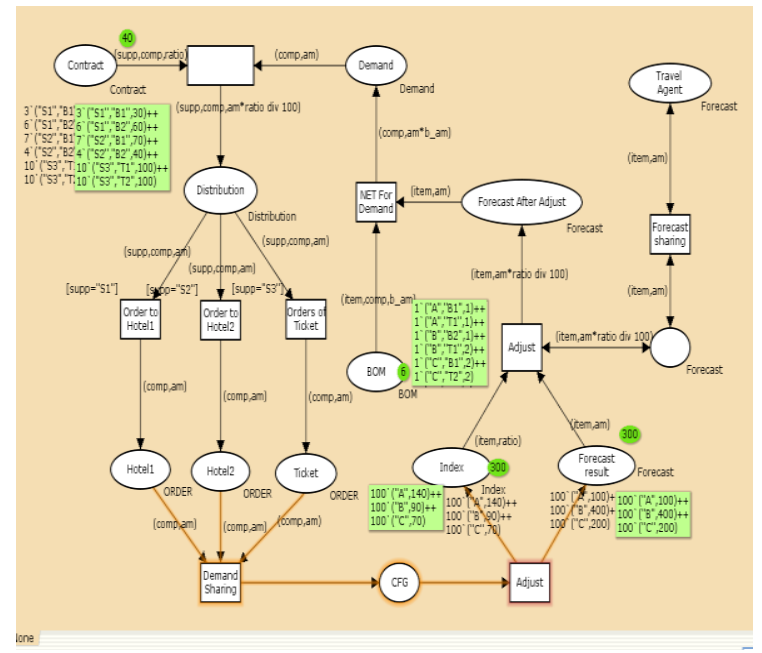

图 7 仿真初始图

在此为了能解决需求预测在多种情况下出现的可能性 不同, 在此所有令牌都尽可能增大, 从而提高数据有效利 用的几率，使得每种可能性都可以发生，以利于效果判断。

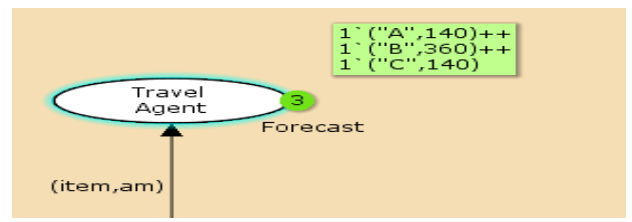

图 8 旅行社需求预测结果

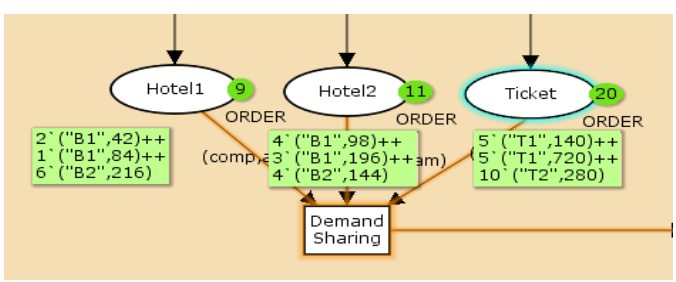

图 9 供应商需求预测结果

4.3 结论

图 8 和图 9 是旅游供应链上的各个主体的预测结果, 对比表 3 与表 4 , 通过观察取值以及整个预测流程可以得出 以下结论:

1) 此仿真链接了旅游供应链上的各个支点, 从旅行社 到酒店、景点, 有助于信息的协同; 需求预测协同小组的 成立能保障需求信息的有效合理沟通、对竞争环境以及突 发事件的合理反馈研究, 有助于协同决策而提高预测精准
度。

2) 在提供足量令牌的前提下, 旅行社得到了经过需求 预测协调小组调整后的预测结果, 各个酒店、景区也相应 得到了依据 BOM 清单、合约约定而计算出来的需求预测结 果。

3）旅游供应链的协同需求预测是可行的。此仿真实现 了需求预测和信息共享, 在旅游供应链中各个支点都得到 了相应的预测结果, 表现出旅游供应链有效协同需求预测。

4）调节在仿真中无法实现，因为这是旅游供应链协同 需求预测中最为复杂的工作, 需要大量的预测方法库和数 据进行深度分析研究, 也需要需求预测协同小组的参与。 此仿真是基于调整结果是已知的情况下进行的循环调整。

\section{5. 结论}

旅游供应链中各个节点都有各自相应的需求所在, 各 个企业的协同可以在信息共享的基础上，对供应链联盟外 的竞争对手、政策环境等影响因素进行深度研究, 通过各 企业对需求预测结果的反馈进行调整, 从而获得更精准的 需求预测结果。本文构建了旅游供应链的协同需求预测流 程仿真框架并分析了协同预测的运作流程。

通过基于 Petri 网的 CPN Tools 对流程构架进行仿真分 析。在证明仿真的可行性的基础上，同时也验证了合理的 信息沟通与协调, 有效的反馈对于精确需求预测的重要性。

对于旅游供应链的协同需求预测仿真例化和应用仍然 需要深度研究与细化, 而且本文的需求预测仿真中没有考 虑时间等变量, 这些都需要后续工作进行研究探讨。

\section{参考文献(References)}

[1] H. Song, X. Zhang, and S. F. Witt, "Collaborative forecasting for tourism supply chain via the Internet", Paper presented at the 18th international symposium on forecasting, pp. 22-25, 2008, Nice, France.

[2] M. Kurtuluş, S. Ulku, and L.B. Toktay, "The value of collaborative forecasting in supply chains", Manufacturing \& Service Operations Management, vol.14, no.1, pp. 82-98, 2012.

[3] L. Amodeo and F. Chu, "Modeling and performance evaluation of supply chains using batch deterministic and stochastic Petri Nets", IEEE Transactions on Automation Science and Engineering, vol. 2, no. 2, pp.132-144, 2005.

[4] Z. Zhang, B. Xi, and H. Yan, "Petri Net-based modeling and analysis of collaborative demand forecasting process", Industrial Engineering Journal, vol. 12, no. 6, pp. 47-51, 2009.

[5] L. Zhu, R. Sui, and Y. Kong, "Simulation based performance analysis in CPN Tools", Microcomputer Applications, vol. 4, no. 10, pp. 29-38, 2008. 